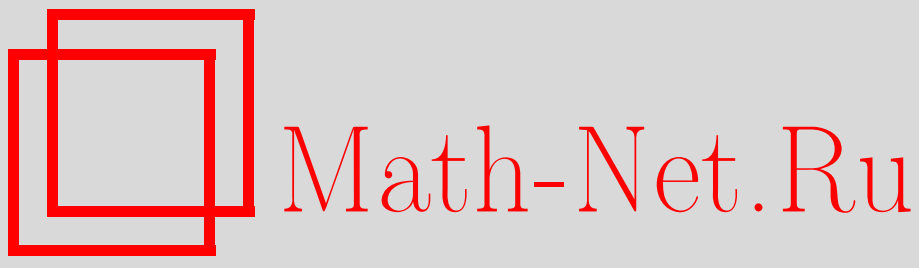

Ю. А. Макаричев, А. В. Стариков, И. С. Ткаченко, Синтез системы подчиненного регулирования электромагнитным подвесом ротора, Вестн. Сам. гос. техн. унта. Сер. Физ.-мат. науки, 2007, выпуск 1(), 143-148

DOI: https://doi.org/10.14498/vsgtu503

Использование Общероссийского математического портала Math-Net.Ru подразумевает, что вы прочитали и согласны с пользовательским соглашением

http://www . mathnet.ru/rus/agreement

Параметры загрузки:

IP : 54.89 .56 .158

26 апреля 2023 г., 13:45:45

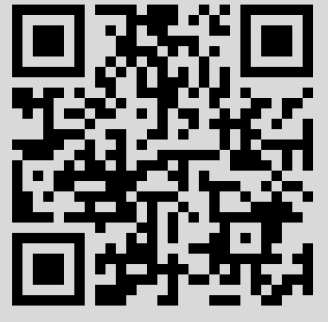




\section{СИНТЕЗ СИСТЕМЫ ПОДЧИНЕННОГО РЕГУЛИРОВАНИЯ ЭЛЕКТРОМАГНИТНЫМ ПОДВЕСОМ РОТОРА}

Рассмотрена система управления электромагнитным подвесом ротора. Проведен параметрический синтез регуляторов по методике систем подчиненного регулирования. Найдены передаточные функции системы управления электромагнитным подвесом ротора по отношению к управляющему и основному возмущающему воздействиям. Построены переходные процессы и частотные характеристики синтезируемой системы. Проанализировано влияние положительной обратной связи по перемещению на работоспособность электромагнитного подвеса ротора.

Применение электромагнитного подвеса ротора в конструкции мощных электродвигателей, насосов, турбин, а также в узлах космической техники позволяет достичь больших скоростей вращения, более продолжительного срока службы и расширить эксплутационные возможности создаваемых агрегатов. Создание системы управления электромагнитным подвесом ротора, отвечающей условиям высокого быстродействия как при отработке возмущающих, так и управляющих воздействий, обладающей высокими свойствами демпфирования вибраций, возникающих в результате действия переменных нагрузок, является актуальной задачей.

Отличительная особенность электромагнитного подшипника (например, радиального) как объекта управления заключается в колебательном характере переходных процессов, наличии положительной обратной связи по перемещению и нелинейности. Тем не менее, линеаризованную математическую модель [1] одной оси радиального электромагнитного подшипника можно представить в виде следующей структурной схемы (рис. 1).

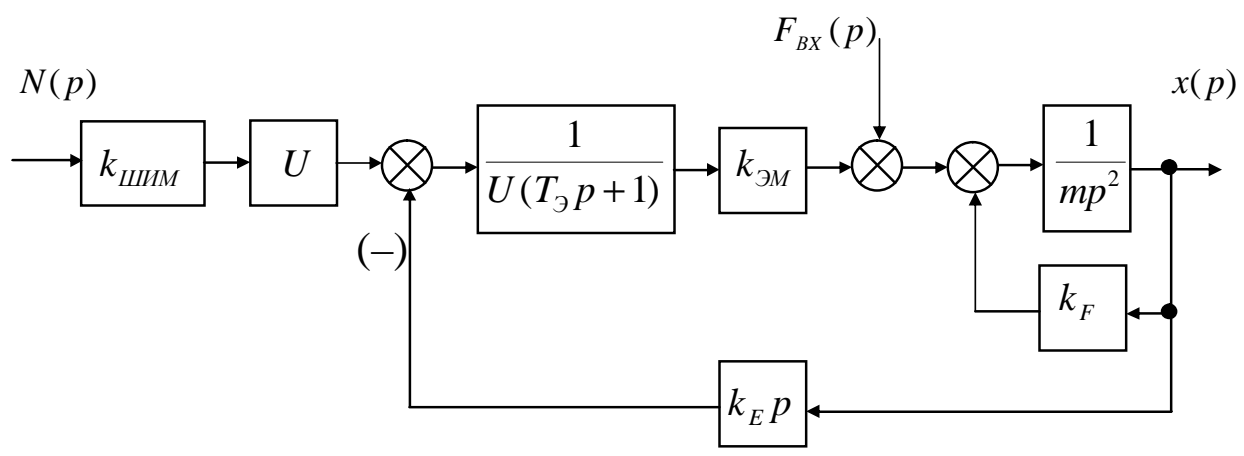

Р и с. 1. Линеаризованная структурная схема одной оси радиального электромагнитного подшипника

Здесь $k_{\text {шим }}-$ коэффициент передачи широтно-импульсного модулятора; $U-$ опорное напряжение широтно-импульсной модуляции; $T_{\ni}=\frac{L}{R}-$ электромагнитная постоянная времени обмоток электромагнитов; $L-$ индуктивность, а $R$ - активное сопротивление обмоток электромагнитов; $k_{\ni м}$ - коэффициент передачи, связывающий приращение силы притяжения магнитов с изменением соотношения токов в их обмотках; $m$ - масса ротора, приходящаяся на один радиальный электромагнитный подшипник; $k_{F}$ - коэффициент положительной обратной связи, учитывающий изменение силы притяжения электромагнитов при смещении ротора от центрального положения; $k_{E}-$ коэффициент, связывающий наводимую в обмотках электромагнитов э.д.с. со скоростью перемещения ротора в магнитном поле. Структурная схема получена в предположении идентичности обмоток электромагнитов, малого отклонения ротора от центрального положения и принятого закона управления токами электромагнитов с помощью широтно-импульсного преобразователя, при котором увеличение тока в одном электромагните приводит к соответствующему уменьшению тока в другом электромагните; $N$ величина сигнала на входе широтно-импульсного модулятора; $x$ - перемещение ротора в поле 
электромагнитов (от центрального положения); $F_{\mathrm{BX}}-$ составляющая внешней возмущающей силы по оси $x$.

Передаточная функция одной оси радиального электромагнитного подшипника по отношению к управляющему воздействию в этом случае

$$
W_{\mathrm{Oу}}^{+}(p)=\frac{\Delta x(p)}{\Delta N(p)} \frac{k_{\text {шим }} k_{\text {Эм }}}{k_{F}\left[\frac{m T_{\ni}}{k_{F}} p^{3}+\frac{m}{k_{F}} p^{2}+\left(\frac{k_{\ni M} k_{E}}{k_{F} U}-T_{\ni}\right) p-1\right]} .
$$

Известны различные подходы к построению систем управления электромагнитным подвесом ротора. Например, применяются одноконтурные системы [2], замкнутые по датчику, бесконтактно измеряющему положение ротора в магнитном поле подшипника. Кроме того, для синтеза систем управления электромагнитным подвесом используют принципы построения многоконтурных систем с одной измеряемой координатой [3]. В любом случае при создании систем управления электромагнитным подвесом ротора преследуют цель обеспечения высокого быстродействия, точности поддержания в центральном положении ротора машины, демпфирования возможных вибраций.

Рассмотрим возможность создания новых систем управления электромагнитным подвесом ротора, построенных по принципам систем подчиненного регулирования координат. Применение такого подхода требует выделения в объекте управления ряда координат, по которым будут замкнуты соответствующие контуры регулирования. Синтезируем двухконтурную систему подчиненного регулирования электромагнитным подшипником с внутренним контуром скорости и внешним контуром положения ротора в поле электромагнитов (рис. 2).

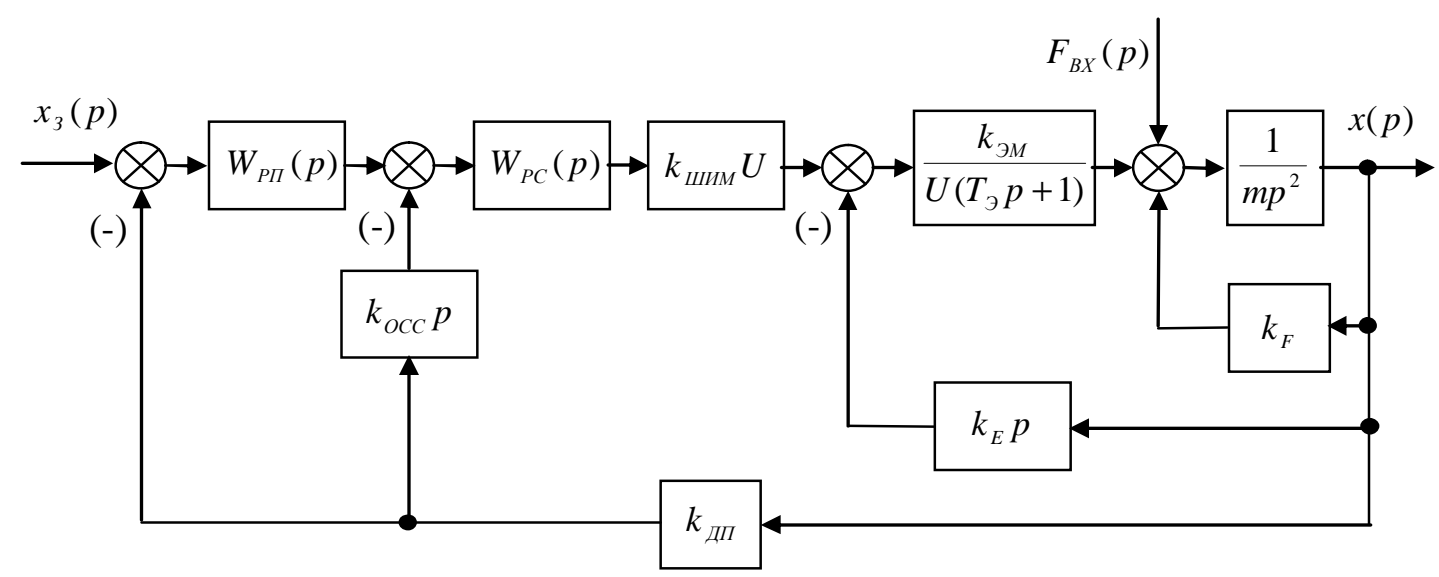

Р и с. 2. Структурная схема одного канала системы подчиненного регулирования электромагнитного подвеса ротора

В качестве датчика главной обратной связи используем бесконтактный датчик с коэффициентом передачи $k_{\text {дп }}$, измеряющий положение ротора в поле электромагнитов. Для выделения сигнала обратной связи по скорости перемещения продифференцируем сигнал датчика положения ротора и введем его в систему с коэффициентом передачи $k_{\mathrm{OCC}}$

В соответствии с общей методикой синтеза регуляторов систем подчиненного регулирования [4] пренебрежем положительной обратной связью по перемещению с коэффициентом передачи $k_{F}$, оценивая впоследствии ее влияние на статические и динамические свойства проектируемой системы. В связи с этим упрощенная структурная схема одного канала системы управления электромагнитным подвесом ротора примет вид, приведенный на рис. 3.

Передаточная функция одной оси радиального электромагнитного подшипника как объекта управления по отношению к управляющему воздействию без учета положительной обратной связи представляет собой интегро-колебательное звено

$$
W_{\text {Оу }}(p)=\frac{k_{\text {шим }} U}{k_{E}\left[\frac{m U}{k_{E} k_{\ni м}} T_{\ni} p^{2}+\frac{m U}{k_{E} k_{\ni м}} p+1\right] p} .
$$




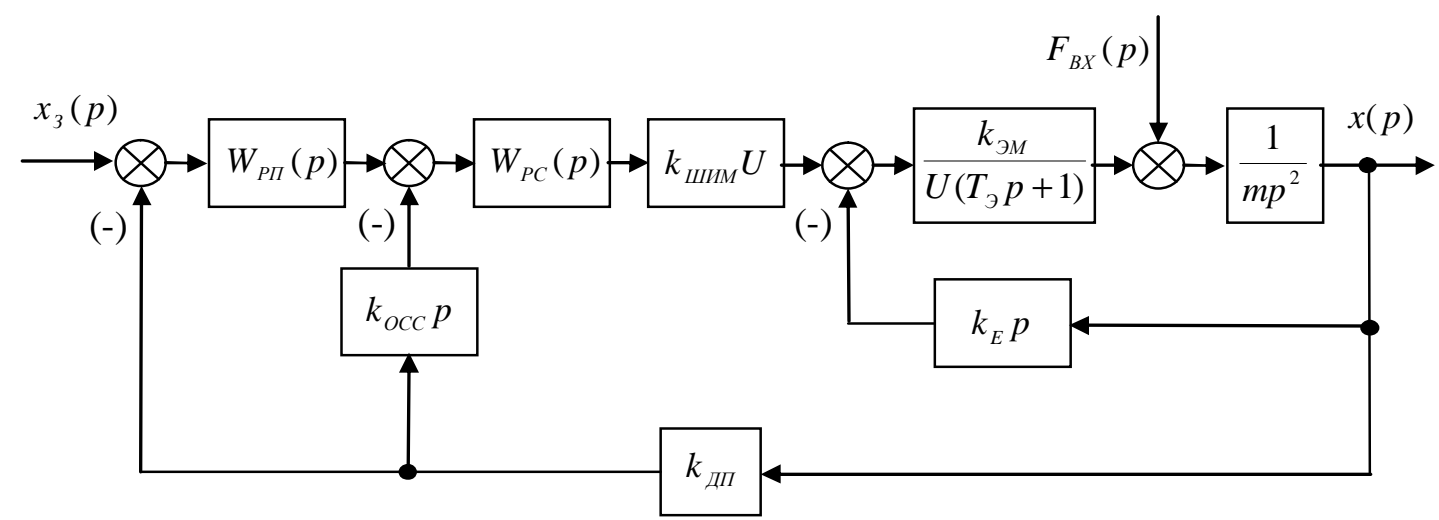

Р и с. 3. Упрощенная структурная одного канала системы подчиненного регулирования электромагнитного подвеса ротора

Как для контура скорости (внутреннего), так и для контура положения (внешнего) используем стандартную настройку на технический оптимум, обладающую высоким быстродействием и малым перерегулированием. Желаемая передаточная функция разомкнутого контура скорости в этом случае

$$
W_{\text {РЖ1 }}(p)=\frac{1}{2 T_{\mu} p\left(T_{\mu} p+1\right)},
$$

где $T_{\mu}$ - малая постоянная времени, инерционность которой не требуется компенсировать. Действительная передаточная функция разомкнутого контура регулирования скорости

$$
W_{\text {РА } 31}(p)=\frac{W_{\text {РС }}(p) k_{\text {шим }} U k_{\text {ДП }} k_{\text {ОСС }}}{k_{E}\left[\frac{m U}{k_{E} k_{\text {ЭМ }}} T_{\ni} p^{2}+\frac{m U}{k_{E} k_{\text {ЭМ }}} p+1\right]} .
$$

Приравнивая выражения (1) и (2), найдем передаточную функцию $W_{\mathrm{PC}}(p)$ регулятора скорости, доставляющую технический оптимум замкнутому контуру:

$$
W_{\mathrm{PC}}(p)=\frac{\left(\frac{m U}{k_{E} k_{\ni \mathrm{M}}} T_{\ni} p^{2}+\frac{m U}{k_{E} k_{\ni \mathrm{M}}} p+1\right)}{2 k_{1} T_{\mu} p\left(T_{\mu} p+1\right)},
$$

где $k_{1}=\frac{U k_{\text {шим }} k_{\text {дП }} k_{\mathrm{OCC}}}{k_{E}}$.

Таким образом, в качестве регулятора скорости в системе подчиненного регулирования электромагнитного подвеса ротора должен быть использован пропорционально-интегральнодифференциальный регулятор с апериодическим фильтром на выходе. Причем, постоянная времени апериодического фильтра равна малой постоянной времени $T_{\mu}$, величину которой можно выбирать произвольно, исходя из требуемого быстродействия системы. Техническая реализация такого регулятора возможна как средствами аналоговой техники на операционном усилителе, так и программно на микропроцессорном устройстве.

Передаточная функция внутреннего замкнутого контура

$$
W_{\text {ЗАМ } 1}(p)=\frac{1}{k_{\text {дП }} k_{\text {ОСС }}\left(2 T_{\mu}^{2} p^{2}+2 T_{\mu} p+1\right) p} .
$$

В соответствии с методикой синтеза систем подчиненного регулирования аппроксимируем ее следующим динамическим звеном

$$
W_{\text {ЗАМ } 1}(p) \approx \frac{1}{k_{\text {дП }} k_{\mathrm{OCC}}\left(2 T_{\mu} p+1\right) p} .
$$

Желаемая передаточная функция разомкнутого контура положения имеет вид

$$
W_{\text {Рж2 }}(p)=\frac{1}{2 T_{\mu 2} p\left(T_{\mu 2} p+1\right)} .
$$

Действительная передаточная функция разомкнутого контура положения с учетом (3) 


$$
W_{\mathrm{PA} 32}(p)=\frac{W_{\mathrm{P \Pi}}(p)}{k_{\mathrm{OCC}}\left(2 T_{\mu} p+1\right) p} .
$$

Приравнивая выражения (4) и (5) и полагая за малую постоянную времени контура положения $T_{\mu 2}=2 T_{\mu}$, найдем передаточную функцию $W_{\text {РП }}(p)$ регулятора положения, доставляющую технический оптимум замкнутому внешнему контуру:

$$
W_{\mathrm{P \Pi}}(p)=k_{\mathrm{P \Pi}}=\frac{k_{\mathrm{OCC}}}{4 T_{\mu}} .
$$

Следовательно, для настройки внешнего контура положения на технический оптимум требуется пропорциональный регулятор с коэффициентом передачи в соответствии с выражением (6). Техническая реализация пропорционального регулятора опять же возможна как средствами аналоговой, так и цифровой техники.

Передаточная функция одного канала замкнутой системы управления электромагнитного подвеса ротора (без учета положительной обратной связи по перемещению) по отношению к управляющему воздействию $x_{3}(p)$

$$
W_{\text {ЗАМ } 2}(p)=\frac{x(p)}{x_{3}(p)}=\frac{1}{k_{\text {дП }}\left(8 T_{\mu}^{3} p^{3}+8 T_{\mu}^{2} p^{2}+4 T_{\mu} p+1\right)} .
$$

Быстродействие системы при отработке управляющего воздействия определяется полностью величиной малой постоянной времени $T_{\mu}$.

Для электромагнитного подвеса ротора с характеристиками: $k_{E}=1461 \mathrm{Bc} / \mathrm{m} ; k_{\ni м}=1306 \mathrm{H}$; $k_{F}=1315900 \mathrm{H} / \mathrm{M} ; \quad m=18$ кг; $R=117,7$ Ом; $L=4,5$ Гн; $T_{\ni}=0,038233 \mathrm{c} ; \quad U=57,7 \mathrm{~B}$; $k_{\text {шим }}=0,001961 ; k_{\text {ОСС }}=1 \mathrm{c}^{-1} ; k_{\text {дП }}=1000000$ дискрет $/ \mathrm{M} ; T_{\mu}=0,0001 \mathrm{c},-$ проведено моделирование системы управления в пакете Matlab Simulink. Переходный процесс по управляющему воздействию (без учета положительной обратной связи) приведен на рис. 4. Качество регулирования определяется высоким быстродействием (время переходного процесса $t_{\text {ПП }}=0,00148 \mathrm{c}$ ) и малым перерегулированием ( $\sigma=7 \%)$.

Передаточная функция системы управления по отношению к возмущению $F_{\mathrm{BX}}(p)$ (без учета положительной обратной связи по перемещению) записывается в виде

$$
W_{\mathrm{B}}(p)=\frac{x(p)}{F_{\mathrm{BX}}(p)}=-\frac{\frac{8 U T_{\mu}^{2}}{k_{E} k_{\ni \mathrm{M}}}\left(T_{\mu} p+1\right)\left(T_{\ni} p+1\right) p}{\left\{\begin{array}{l}
\frac{8 m U T_{\ni} T_{\mu}^{3}}{k_{E} k_{Э \mathrm{M}}} p^{5}+\frac{8 m U T_{\mu}^{2}\left(T_{\ni}+T_{\mu}\right)}{k_{E} k_{Э \mathrm{M}}} p^{4}+\left[8 T_{\mu}^{3}+\frac{4 m U T_{\mu}\left(T_{\ni}+T_{\mu}\right)}{k_{E} k_{Э \mathrm{M}}}\right] p^{3}+ \\
+\left[8 T_{\mu}^{2}+\frac{m U\left(T_{\ni}+T_{\mu}\right)}{k_{E} k_{\text {ЭМ }}}\right] p^{2}+\left(4 T_{\mu}+\frac{m U}{k_{E} k_{Э \mathrm{M}}}\right) p+1
\end{array}\right\}} .
$$

Знак «-» в выражении (7) учитывает то обстоятельство, что основным возмущением является сила тяжести, направленная в отрицательном направлении относительно осей электромагнитного подшипника.

Особый интерес представляет исследование частотной характеристики электромагнитного подшипника по отношению к возмущающему воздействию. В соответствии с передаточной функцией (7) можно получить выражение для частотной характеристики

$$
W(j \omega)=P(\omega)+j Q(\omega),
$$

где $P(\omega)=\frac{\left(b_{2} \omega-b_{0} \omega^{3}\right)\left(a_{0} \omega^{5}-a_{2} \omega^{3}+a_{4} \omega\right)-b_{1} \omega^{2}\left(a_{1} \omega^{4}-a_{3} \omega^{2}+1\right)}{\left(a_{1} \omega^{4}-a_{3} \omega^{2}+1\right)^{2}+\left(a_{0} \omega^{5}-a_{2} \omega^{3}+a_{4} \omega\right)^{2}}$;

$$
Q(\omega)=\frac{\left(b_{2} \omega-b_{0} \omega^{3}\right)\left(a_{1} \omega^{4}-a_{3} \omega^{2}+1\right)+b_{1} \omega^{2}\left(a_{0} \omega^{5}-a_{2} \omega^{3}+a_{4} \omega\right)}{\left(a_{1} \omega^{4}-a_{3} \omega^{2}+1\right)^{2}+\left(a_{0} \omega^{5}-a_{2} \omega^{3}+a_{4} \omega\right)^{2}}
$$$$
b_{0}=\frac{8 U T_{\ni} T_{\mu}^{3}}{k_{E} k_{\ni \mathrm{M}}} ; b_{1}=\frac{8 U T_{\mu}^{2}\left(T_{\ni}+T_{\mu}\right)}{k_{E} k_{\ni \mathrm{M}}} ; b_{1}=\frac{8 U T_{\mu}^{2}}{k_{E} k_{\ni \mathrm{M}}} ; a_{0}=\frac{8 m U T_{\ni} T_{\mu}^{3}}{k_{E} k_{\ni \mathrm{M}}} ; a_{1}=\frac{8 m U T_{\mu}^{2}\left(T_{\ni}+T_{\mu}\right)}{k_{E} k_{\ni \mathrm{M}}} ;
$$$$
a_{2}=8 T_{\mu}^{3}+\frac{4 m U T_{\mu}\left(T_{\ni}+2 T_{\mu}\right)}{k_{E} k_{\ni \mathrm{M}}} ; a_{3}=8 T_{\mu}^{2}+\frac{m U\left(T_{\ni}+4 T_{\mu}\right)}{k_{E} k_{\ni \mathrm{M}}} ; a_{4}=4 T_{\mu}+\frac{m U}{k_{E} k_{\ni \mathrm{M}}} .
$$ 
При выбранных параметрах электромагнитов, широтно-импульсного модулятора и регуляторов логарифмическая амплитудно-частотная характеристика радиального электромагнитного подшипника принимает вид, показанный на рис. 5. Анализ рассматриваемой частотной характеристики по отношению к возмущающему воздействию $F_{\mathrm{BX}}(p)$ показывает, что в системе наблюдается значительное ослабление периодических возмущений, следовательно, и вибраций. Максимальное колебание ротора будет наблюдаться на частоте 560 рад/с (89 Гц), при этом коэффициент ослабления амплитуды составляет 154 дБ. В остальном частотном диапазоне коэффициент ослабления амплитуд еще больше. Например, на частоте 314 рад/с (50 Гц) коэффициент ослабления - 160 дБ.

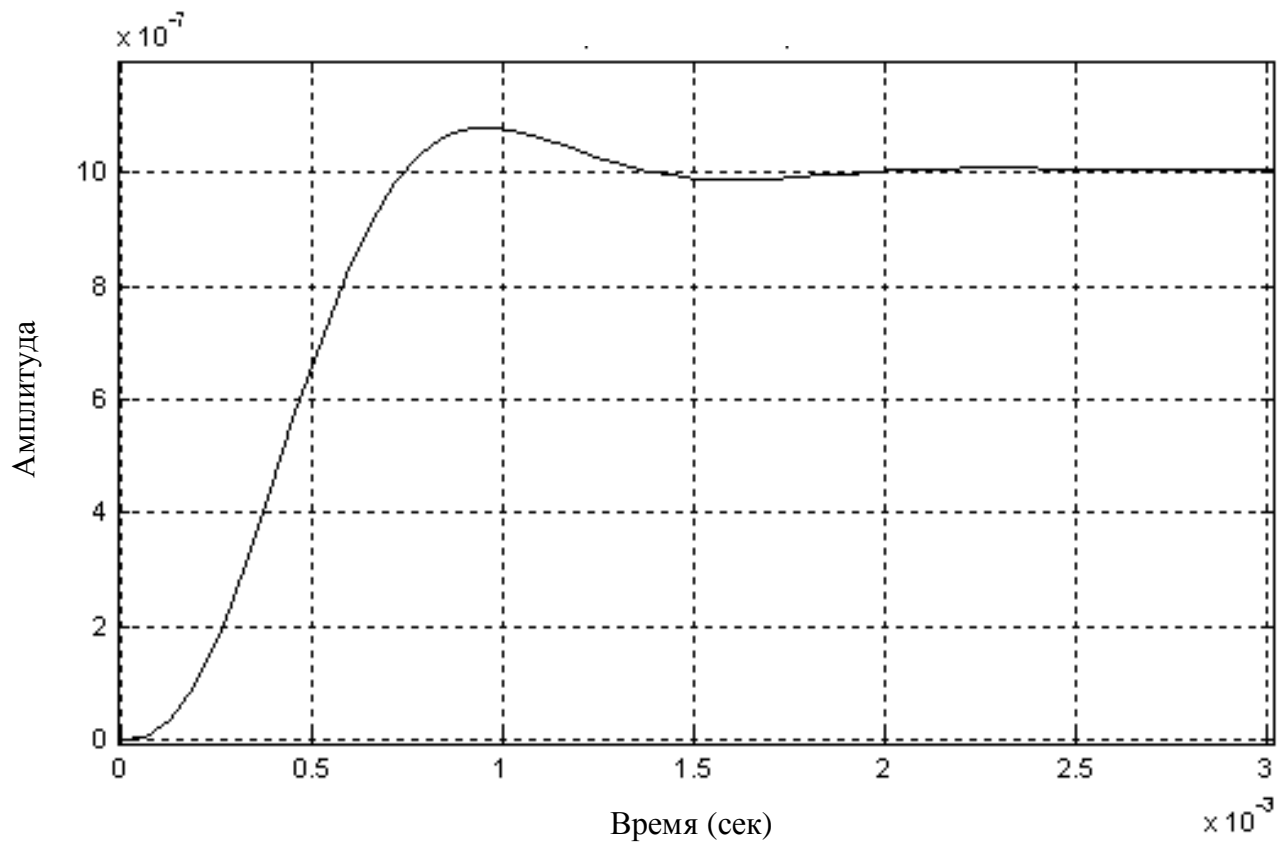

Р и с. 4. Переходный процесс по управляющему воздействию (без учета положительной обратной связи по перемещению)

Приведенные выше результаты по выбору регуляторов, определению передаточных функций замкнутой системы и, следовательно, статических и динамических характеристик электромагнитного подвеса ротора получены в пренебрежении положительной обратной связью по перемещению, поэтому необходимо оценить ее влияние. Для этого при выбранных типах и параметрах регуляторов найдем передаточную функцию замкнутой системы управления электромагнитного подвеса ротора по отношению к управляющему воздействию $x_{3}(p)$ с учетом положительной обратной связи по перемещению

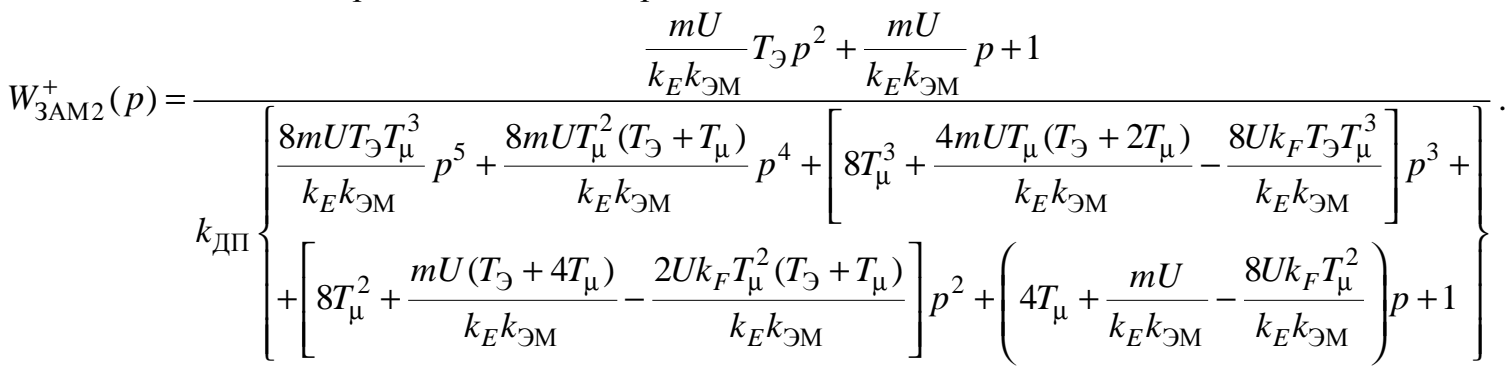

Переходный процесс, построенный по последней передаточной функции, показывает, что показатели качества регулирования практически совпадают с аналогичными результатами, полученными без учета положительной обратной связи по перемещению (рис. 4).

Подводя итог сказанному, следует отметить, что синтезированная система подчиненного регулирования электромагнитным подвесом ротора обладает высоким быстродействием, астатизмом по отношению к основным возмущениям и хорошими демпфирующими свойствами к внешним вибрациям. 


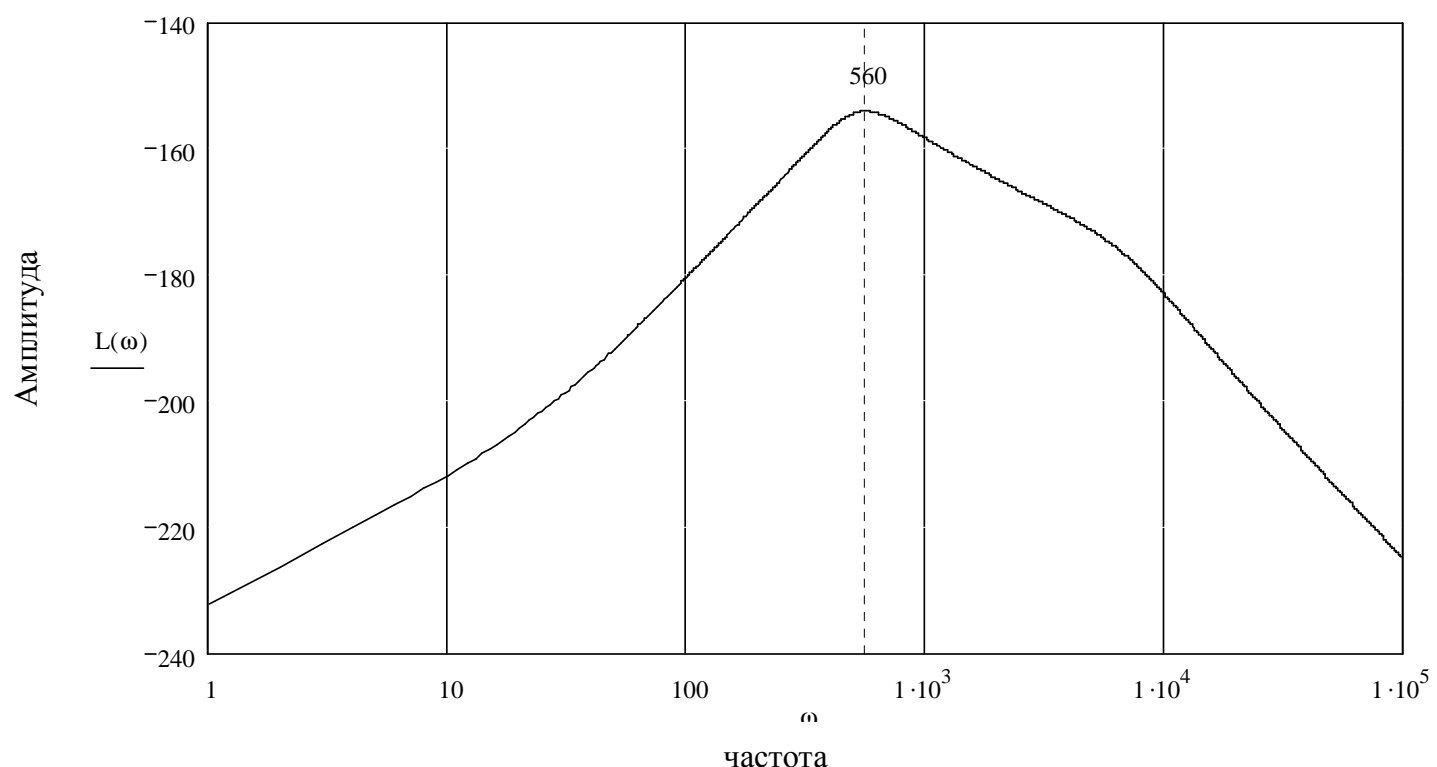

Р и с. 5. Логарифмическая амплитудно-частотная характеристика радиального электромагнитного подшипника по отношению к возмущающему воздействию $F_{\mathrm{BX}}(p)$

\section{БИБЛИОГРАФИЧЕСКИЙ СПИСОК}

1. Макаричев Ю. А., Стариков А. В., Стариков А. В. Математическая модель радиального электромагнитного подшипника как объекта управления // Электротехнические системы и комплексы: Межвузовский сборник науч. трудов. Магнитогорск: МГТУ, 1998. С. 80-86.

2. Вейнберг Д. М., Верещагин В. П. К определению основных параметров электромагнитных подшипников. М.: Труды ВНИИЭМ, Т. 89, 1989. С. 12-19.

3. Патент России № 2181922. Система управления электромагнитным подвесом ротора / Ю. А. Макаричев, А. B. Стариков, А. В. Стариков. Опубл. 27.04.2002. Бюл. № 12.

4. Panonopm Э. Я. Системы подчиненного регулирования электроприводов постоянного тока. Куйбышев: КПтИ, 1985. $56 \mathrm{c}$.

Поступила 4.07.2006 г.

\section{УДК 666.92.041}

\section{B. В. Копцев}

\section{МЕТОДИКА РАСЧЕТА ГОРЕЛОЧНЫХ УСТРОЙСТВ НА БАЗЕ СОПЛА С ЦЕНТРАЛЬНЫМ ТЕЛОМ}

Приведена методика расчета горения высокоскоростных потоков газа с использованием горелочного устройства на базе сопла с иентральным телом, схема головки которого подобна аэродинамической конфигурации кольцевого сопла с нулевым наклоном минимального сечения с двойным расширением. Представлены конструктивные особенности серии горелочных устройств с центральным телом, разработанных автором, и проиллюстрированы их основные достоинства.

Сопла с центральным телом (или «тарельчатые» сопла, сопла с прямолинейной верхней стенкой) находят, в основном, применение в авиапромышленности при изготовлении реактивных двигателей [1-5]. Автором предложена еще одна область практического применения сопел с центральным телом в качестве одного из элементов конструкции горелочных устройств в нагревательных и термических печах различного назначения [6-9]. 